This item was submitted to Loughborough's Research Repository by the author.

Items in Figshare are protected by copyright, with all rights reserved, unless otherwise indicated.

\title{
Soft core fluid in a quenched matrix of soft core particles: a mobile mixture in a model gel
}

\section{PLEASE CITE THE PUBLISHED VERSION}

http://dx.doi.org/10.1103/PhysRevE.73.011506

\section{PUBLISHER}

(C) The American Physical Society

\section{VERSION}

VoR (Version of Record)

\section{PUBLISHER STATEMENT}

This work is made available according to the conditions of the Creative Commons Attribution-NonCommercialNoDerivatives 4.0 International (CC BY-NC-ND 4.0) licence. Full details of this licence are available at: https://creativecommons.org/licenses/by-nc-nd/4.0/

\section{LICENCE}

CC BY-NC-ND 4.0

\section{REPOSITORY RECORD}

Archer, Andrew J., Matthias Schmidt, and Robert Evans. 2019. "Soft Core Fluid in a Quenched Matrix of Soft Core Particles: A Mobile Mixture in a Model Gel”. figshare. https://hdl.handle.net/2134/16038. 


\title{
Soft core fluid in a quenched matrix of soft core particles: A mobile mixture in a model gel
}

\author{
A. J. Archer, ${ }^{1, *}$ M. Schmidt, ${ }^{1,2}$ and R. Evans ${ }^{1}$ \\ ${ }^{1}$ H.H. Wills Physics Laboratory, University of Bristol, Tyndall Avenue, Bristol BS8 1TL, United Kingdom \\ ${ }^{2}$ Institut für Theoretische Physik II, Heinrich-Heine-Universität Düsseldorf, Universitätsstraße 1, D-40225 Düsseldorf, Germany
}

(Received 26 September 2005; published 30 January 2006)

\begin{abstract}
We present a density-functional study of a binary phase-separating mixture of soft core particles immersed in a random matrix of quenched soft core particles of larger size. This is a model for a binary polymer mixture immersed in a cross-linked rigid polymer network. Using the replica "trick" for quenched-annealed mixtures we derive an explicit density functional theory that treats the quenched species on the level of its one-body density distribution. The relation to a set of effective external potentials acting on the annealed components is discussed. We relate matrix-induced condensation in bulk to the behavior of the mixture around a single large particle. The interfacial properties of the binary mixture at a surface of the quenched matrix display a rich interplay between capillary condensation inside the bulk matrix and wetting phenomena at the matrix surface.
\end{abstract}

DOI: 10.1103/PhysRevE.73.011506

PACS number(s): 78.55.Mb, 61.20.Gy, 64.10.+h

\section{INTRODUCTION}

Spatial confinement can have a dramatic effect on the properties of a fluid. The combined effects of finite pore size and substrate-fluid interactions can alter the location of phase boundaries from what is found for the bulk adsorbate. Many studies have focused on the equilibrium structure and phase behavior of fluids confined in model pores or capillaries and phenomena such as capillary condensation (the shifted gasliquid transition) and capillary freezing are rather well understood for a one-component fluid confined in a single, infinitely long slit or cylinder $[1,2]$. The kinetics of nucleation accompanying capillary condensation has also been investigated [3]. When the adsorbate consists of more than one species, capillary-induced fluid-fluid demixing can occur in addition to capillary condensation-see, e.g., [4] for recent work. However, most porous media exhibit complicated, interconnected pore shapes [2] and cannot be modelled as idealised capillaries. Rather, a random porous network structure provides a more realistic starting model. In recent years the direct treatment of fluids adsorbed random pore structures has been achieved within the framework of lattice fluids yielding significant new insight into adsorption phenomena [5-10].

An alternative way of modeling porous structures is to consider pore structures generated by quenched configurations of model fluids. These configurations carry a statistical weight that is determined solely by the Hamiltonian of the (pure) model fluid. The adsorbate fluid is then brought into contact with the quenched particles (referred to as the matrix) and is "annealed" in the sense that it is under the influence of the matrix as an external potential. In addition to the ensemble average, an average over all realizations of the matrix must be carried out. Frequently such models are tackled using liquid state integral equation theory, relying on the replica Ornstein-Zernike equations [11,12] —an approach that works on the level of two-body correlation functions.

\footnotetext{
*Andrew.Archer@bristol.ac.uk
}

Recently a classical density functional theory (DFT) $[13,14]$, operating on the one-body level, was shown to be able to treat consistently quenched-annealed mixtures. This quenched-annealed DFT (or replica DFT) $[15,16]$ treats both the annealed and the quenched species on the level of their one-body density distributions. Hence the complicated external potential that the quenched particles exert on the fluid never enters explicitly into the theoretical framework. This approach constitutes an enormous simplification as far as practical computations are concerned. Investigations were carried out for hard core models and for the quenchedannealed Asakura-Oosawa-Vrij model of colloid-polymer mixtures [17].

The Gaussian core model (GCM) [18] belongs to a different class of system, characterized by fully penetrable pair interactions, i.e., at zero spatial separation between the centers of a pair of particles the potential energy is finite and is comparable in magnitude to the thermal energy. The fact that a very simple density functional approximation is accurate at high fluid densities [18-23] has facilitated a number of detailed investigations and much is known about the bulk and interfacial properties, as well as subtle solvation phenomena, in this model fluid [21,22,24-27].

In the present work, we are concerned with a quenchedannealed GCM modeling a binary polymer solution as an adsorbate in a quenched (cross-linked) rigid polymer network, i.e., a mobile binary mixture adsorbed in a model gel. Quenching components of a GCM mixture results in soft confinement of the annealed species, as opposed to the hard confinement considered with replica DFT in previous studies. The particularly simple [random phase approximation (RPA)] form of the excess free energy functional enables us to elucidate the structure of the replica DFT. We can relate the effect of the quenched component directly to the influence of an effective external potential acting on the fluid particles; this is shown to take the form of a convolution of the matrix one-body density profile and the matrix-fluid (pair) interaction potential.

The paper is organized as follows. In Sec. II we give a brief overview of the statistical mechanics of quenchedannealed mixtures. In Sec. III the DFT for the quenched- 
annealed GCM is presented. The case of matrices with uniform density is covered in Sec. IV. We present results for bulk properties of a particular mixture in Sec. V and treat inhomogeneities induced by the surface of a matrix in Sec. VI. Discussion and conclusions are presented in Sec. VII.

\section{QUENCHED-ANNEALED SYSTEMS}

We consider a system composed of three different species of particles. One of the species, labelled 0 , is quenched, forming a matrix into which the remaining two species, labelled 1 and 2, are annealed, i.e., the particles of species 0 form an immobile network in which the other species are free to move. The correlations between the quenched matrix particles are independent of the adsorbate particles and are determined solely by interactions between matrix particles. We denote particle numbers by $N_{a}$, where $a=0,1,2$ labels the different species, and denote the sets of position coordinates of particles of species $a$ by $\mathbf{r}^{N_{a}} \equiv\left\{\mathbf{r}_{i, a}, i=1, \ldots, N_{a}\right\}$. The total external potential energy of species $a$, the total potential due to pair interactions between particles of species $a$, and the total potential due to pair interactions between particles of (unlike) species $a$ and $b$, are defined, respectively, by

$$
\begin{gathered}
V_{a}\left(\mathbf{r}^{N_{a}}\right)=\sum_{i=1}^{N_{a}} V_{a}^{\mathrm{ext}}\left(\mathbf{r}_{i}\right), \\
\Phi_{a a}\left(\mathbf{r}^{N_{a}}\right)=\sum_{i=1}^{N_{a}} \sum_{j=i+1}^{N_{a}} v_{a a}\left(\left|\mathbf{r}_{i}-\mathbf{r}_{j}\right|\right), \\
\Phi_{a b}\left(\mathbf{r}^{N_{a}}, \mathbf{r}^{N_{b}}\right)=\sum_{i=1}^{N_{a}} \sum_{j=1}^{N_{b}} v_{a b}\left(\left|\mathbf{r}_{i}-\mathbf{r}_{j}\right|\right), \quad a \neq b,
\end{gathered}
$$

where $V_{a}^{\mathrm{ext}}\left(\mathbf{r}_{i}\right)$ is the external potential acting on particle $i$ of species $a$ and $v_{a b}\left(\left|\mathbf{r}_{i}-\mathbf{r}_{j}\right|\right)$ is the pair potential between particle $i$ of species $a$ and particle $j$ of species $b$. It is important to note that for quenched-annealed systems the potentials $\Phi_{01}$ and $\Phi_{02}$, exerted by the quenched particles on the annealed matrix particles, never enter into the description of any properties of the matrix (i.e., species 0 ); the latter originate solely from $V_{0}\left(\mathbf{r}^{N_{0}}\right)$ and $\Phi_{00}\left(\mathbf{r}^{N_{0}}\right)$.

Hence, the partition function for the quenched matrix particles is

$$
\Xi_{0}\left(\mu_{0}\right)=\operatorname{Tr}_{N_{0}} \exp \left[-\beta\left(\Phi_{00}+V_{0}\right)\right],
$$

where $\mu_{0}$ is the chemical potential for the quenched particles and the classical trace is

$$
\operatorname{Tr}_{N_{a}}(\cdot)=\sum_{N_{a}=0}^{\infty} \frac{z_{a}^{N_{a}}}{N_{a} !} \int \mathrm{d} \mathbf{r}_{a}^{N_{a}}(\cdot),
$$

where $z_{a}=\Lambda_{a}^{-3} \exp \left(\beta \mu_{a}\right)$ is the fugacity, $\Lambda_{a}$ is the thermal de Broglie wavelength for species $a$, and $\beta=1 / k_{B} T$, where $k_{B}$ is the Boltzmann constant. Strictly, $\Xi_{0}=\Xi_{0}\left(\mu_{0}, T, V\right)$ is a function of the chemical potential $\mu_{0}$, the temperature $T$, and the volume of the system $V$. However, we will suppress the de- pendence on $T$ and $V$ for notational convenience. The grand potential for the quenched particles is then given by

$$
\Omega_{0}\left(\mu_{0}\right)=-k_{B} T \ln \Xi_{0}\left(\mu_{0}\right) .
$$

For a given configuration of matrix particles, $\mathbf{r}^{N_{0}}$, the grand potential for the annealed particles, adsorbed in the matrix, is

$$
\begin{aligned}
\Xi_{12}\left(\mathbf{r}^{N_{0}}, \mu_{1}, \mu_{2}\right)= & \operatorname{Tr}_{N_{1}} \operatorname{Tr}_{N_{2}} \exp \left[-\beta\left(V_{1}+V_{2}+\Phi_{11}+\Phi_{22}\right.\right. \\
& \left.\left.+\Phi_{12}+\Phi_{01}+\Phi_{02}\right)\right]
\end{aligned}
$$

and the grand potential for this configuration is

$$
\widetilde{\Omega}_{12}\left(\mathbf{r}^{N_{0}}, \mu_{1}, \mu_{2}\right)=-k_{B} T \ln \Xi_{12}\left(\mathbf{r}^{N_{0}}, \mu_{1}, \mu_{2}\right) .
$$

We now average this grand potential over all configurations of quenched matrix particles:

$\Omega_{12}\left(\mu_{0}, \mu_{1}, \mu_{2}\right)=\Xi_{0}^{-1} \operatorname{Tr}_{N_{0}} \widetilde{\Omega}_{12}\left(\mathbf{r}^{N_{0}}, \mu_{1}, \mu_{2}\right) \exp \left[-\beta\left(\Phi_{00}+V_{0}\right)\right]$.

$\Omega_{12}$ is a key quantity: it determines many of the properties of the fluid in the matrix. We now apply the replica "trick" that proves to be a powerful idea to actually calculate $\Omega_{12}$ $[15,16,28]$.

The idea is to introduce $s$ "replicas", or copies, of the sets of particles of species 1 and 2 into the matrix of quenched particles. Particles do not interact with particles of a different replica, but only with particles of the same replica as well as with the matrix particles. The partition sum for this $(2 s+1)$-component mixture is

$$
\begin{aligned}
& \Xi\left(\mu_{0}, \mu_{1}, \mu_{2} ; s\right) \\
& =\operatorname{Tr}_{N_{0}} \exp \left[-\beta\left(\Phi_{00}+V_{0}\right)\right]\left\{\operatorname{Tr}_{N_{1}} \operatorname{Tr}_{N_{2}}\right. \\
& \left.\quad \times \exp \left[-\beta\left(V_{1}+V_{2}+\Phi_{11}+\Phi_{22}+\Phi_{12}+\Phi_{01}+\Phi_{02}\right)\right]\right\}^{s}
\end{aligned}
$$

and the corresponding grand potential follows as

$$
\Omega\left(\mu_{0}, \mu_{1}, \mu_{2} ; s\right)=-k_{B} T \ln \Xi\left(\mu_{0}, \mu_{1}, \mu_{2} ; s\right) .
$$

Performing an analytic continuation in $s$, and noting that $\lim _{s \rightarrow 0} \mathrm{~d} x^{s} / \mathrm{d} s=\ln x$, we find [16]

$$
\Omega_{12}\left(\mu_{0}, \mu_{1}, \mu_{2}\right)=\lim _{s \rightarrow 0} \frac{\partial}{\partial s} \Omega\left(\mu_{0}, \mu_{1}, \mu_{2} ; s\right) .
$$

Thus we have constructed a means of tackling quenchedannealed systems by considering the corresponding replicated (and fully annealed) mixture. At this point we can make connection with DFT, which provides a powerful approach to calculating the grand potential for inhomogeneous systems. In DFT the grand potential is expressed as a functional of the fluid one-body density profiles, $\rho_{a}(\mathbf{r})[13,14]$. The grand potential in (11) is obtained by considering a variational grand potential functional, $\Omega^{\operatorname{var}}\left(\left[\left\{\rho_{a}\right\}\right],\left\{\mu_{a}\right\} ; s\right)$, which is minimized with respect to the density profiles $[13,14]$, 


$$
\frac{\delta \Omega^{\operatorname{var}}\left(\left[\left\{\rho_{a}\right\}\right],\left\{\mu_{a}\right\} ; s\right)}{\delta \rho_{a}(\mathbf{r})}=0 .
$$

Inserting the density profiles that are the solutions to (13) into $\Omega^{\mathrm{var}}$ yields as the minimal value the true grand potential, $\Omega\left(\mu_{0}, \mu_{1}, \mu_{2} ; s\right)$. Performing an analytic continuation in $s$, and Taylor expanding $\Omega^{\mathrm{var}}$ around $s=0$, gives

$$
\begin{aligned}
\Omega^{\operatorname{var}}\left(\left[\left\{\rho_{a}\right\}\right],\left\{\mu_{a}\right\} ; s\right)= & \Omega_{0}^{\operatorname{var}}\left(\left[\rho_{0}\right], \mu_{0}\right)+s \Omega_{12}^{\operatorname{var}}\left(\left[\left\{\rho_{a}\right\}\right],\left\{\mu_{a}\right\}\right) \\
& +\mathcal{O}\left(s^{2}\right) .
\end{aligned}
$$

$\Omega_{0}^{\operatorname{var}}\left(\left[\rho_{0}\right], \mu_{0}\right)$ is simply the grand potential for the pure system of matrix particles; furthermore $\Omega_{12}^{\operatorname{var}}\left(\left[\left\{\rho_{a}\right\}\right],\left\{\mu_{a}\right\}\right)=\lim _{s \rightarrow 0} \partial \Omega^{\operatorname{var}}\left(\left[\left\{\rho_{a}\right\}\right],\left\{\mu_{a}\right\} ; s\right) / \partial s$. The key point is that $\Omega_{12}^{\mathrm{var}}$ in (14) corresponds to an average over the different matrix configurations and is hence only a functional of the matrix density profile $\rho_{0}(\mathbf{r})$, rather than depending on particular matrix configurations $\mathbf{r}^{N_{0}}$. Explicitly, we can write the first functional on the right-hand side of (14) as

$$
\begin{aligned}
\Omega_{0}^{\mathrm{var}}\left(\left[\rho_{0}(\mathbf{r})\right], \mu_{0}\right)= & \mathcal{F}_{0}^{\mathrm{id}}\left[\rho_{0}(\mathbf{r})\right]+\mathcal{F}_{0}^{\mathrm{ex}}\left[\rho_{0}(\mathbf{r})\right] \\
& -\int \mathrm{d} \mathbf{r} \rho_{0}(\mathbf{r})\left[\mu_{0}-V_{0}^{\mathrm{ext}}(\mathbf{r})\right],
\end{aligned}
$$

where

$$
\mathcal{F}_{a}^{\mathrm{id}}\left[\rho_{a}(\mathbf{r})\right]=k_{B} T \int \mathrm{d} \mathbf{r} \rho_{a}(\mathbf{r})\left\{\ln \left[\Lambda_{a}^{3} \rho_{a}(\mathbf{r})\right]-1\right\}
$$

is the Helmholtz free energy of the ideal gas and $\mathcal{F}_{0}^{\mathrm{ex}}\left[\rho_{0}(\mathbf{r})\right]$ is the excess contribution to the total Helmholtz free energy arising from interactions between the matrix particles. Similarly,

$$
\begin{aligned}
\Omega_{12}^{\mathrm{var}} & {\left.\left[\rho_{0}, \rho_{1}, \rho_{2}\right], \mu_{1}, \mu_{2}\right) } \\
= & \mathcal{F}_{1}^{\mathrm{id}}\left[\rho_{1}(\mathbf{r})\right]+\mathcal{F}_{2}^{\mathrm{id}}\left[\rho_{2}(\mathbf{r})\right]+\mathcal{F}_{12}^{\mathrm{ex}}\left[\rho_{0}(\mathbf{r}), \rho_{1}(\mathbf{r}), \rho_{2}(\mathbf{r})\right] \\
& -\sum_{a=1}^{2} \int \mathrm{d} \mathbf{r} \rho_{a}(\mathbf{r})\left[\mu_{a}-V_{a}^{\mathrm{ex}}(\mathbf{r})\right],
\end{aligned}
$$

where $\mathcal{F}_{a}^{\text {id }}\left[\rho_{a}(\mathbf{r})\right], \quad a=1,2$, is given by Eq. (16) and $\mathcal{F}_{12}^{\mathrm{ex}}\left[\rho_{0}, \rho_{1}, \rho_{2}\right]$ is the contribution to the Helmholtz free energy from interactions between the particles. This quantity includes interactions between the annealed particles and the quenched matrix particles. In general, $\mathcal{F}_{12}^{\mathrm{ex}}$ is an unknown functional. However, depending on the form of the interactions between the particles, there exist a number of accurate approximate functionals [14].

The variational principle (13), when applied to (14), becomes $[15,16]$

$$
\begin{gathered}
\frac{\delta \Omega_{0}^{\mathrm{var}}\left(\left[\rho_{0}\right], \mu_{0}\right)}{\delta \rho_{0}(\mathbf{r})}=0, \\
\frac{\delta \Omega_{12}^{\mathrm{var}}\left(\left[\rho_{0}, \rho_{1}, \rho_{2}\right], \mu_{1}, \mu_{2}\right)}{\delta \rho_{a}(\mathbf{r})}=0, \quad a=1,2 .
\end{gathered}
$$

One therefore uses (18), together with (15) and a suitable approximation for $\mathcal{F}_{0}^{\mathrm{ex}}\left[\rho_{0}(\mathbf{r})\right]$, to first calculate the average one-body density for the quenched matrix particles, $\rho_{0}(\mathbf{r})$.
Then one uses this density profile as input to calculate the density profiles $\rho_{1}(\mathbf{r})$ and $\rho_{2}(\mathbf{r})$ of the annealed species using Eqs. (17) and (19), again with a suitable approximation for $\mathcal{F}_{12}^{\mathrm{ex}}\left[\left\{\rho_{a}(\mathbf{r})\right\}\right]$. In the next section we shall describe specific approximations for particles that interact via repulsive Gaussian pair potentials.

\section{DFT FOR THE QUENCHED-ANNEALED GCM}

The interactions between macromolecules such as polymers or dendrimers in solution can be modeled by means of an effective pair potential between the centers of mass of the macromolecules. This implies treating each macromolecule as a (soft) "particle". For a good solvent the effective pair potential is purely repulsive and is well approximated by a repulsive Gaussian form [18,29-35]. In the GCM, the potential between particles of species $a$ and $b$ is given by

$$
v_{a b}(r)=\epsilon_{a b} \exp \left(-r^{2} / R_{a b}^{2}\right),
$$

where $\epsilon_{a b}$ is the energy penalty (due to entropic effects on the segment level) for complete overlap of the centers of mass. Typically $\epsilon_{a b} \sim 2 k_{B} T$ and $R_{a a} \sim R_{g, a}$, the radius of gyration of particles of the $a$ th species. The pair potential parameters for the potentials between unlike species, as obtained from simulations of model polymer solutions [29], are consistent with the (non additive) mixing rules,

$$
R_{12}^{2}=\frac{1}{2}\left(R_{11}^{2}+R_{22}^{2}\right)
$$

and

$$
\epsilon_{12} \lesssim \epsilon_{11}=\epsilon_{22} .
$$

For the GCM the mean-field excess Helmholtz free energy functional,

$$
\mathcal{F}^{\operatorname{ex}}\left[\left\{\rho_{a}(\mathbf{r})\right\}\right]=\frac{1}{2} \sum_{a, b} \int \mathrm{d} \mathbf{r} \int \mathrm{d} \mathbf{r}^{\prime} \rho_{a}(\mathbf{r}) \rho_{b}\left(\mathbf{r}^{\prime}\right) v_{a b}\left(\left|\mathbf{r}-\mathbf{r}^{\prime}\right|\right),
$$

is known to be accurate at high densities [18-23] when each particle interacts with a large number of neighboring particles - the classic mean field situation. The functional (23) generates the RPA closure for the pair direct correlation functions upon differentiation $[14,18]$ since

$$
c_{a b}^{(2)}\left(\mathbf{r}, \mathbf{r}^{\prime}\right) \equiv-\beta \frac{\delta^{2} \mathcal{F}^{\operatorname{ex}}\left[\left\{\rho_{a}(\mathbf{r})\right\}\right]}{\delta \rho_{a}(\mathbf{r}) \delta \rho_{b}\left(\mathbf{r}^{\prime}\right)}=-\beta v_{a b}\left(\left|\mathbf{r}-\mathbf{r}^{\prime}\right|\right) .
$$

Here we focus on the case of a ternary mixture where one species of GCM particles is quenched (treating cases with larger numbers of both annealed and quenched components requires straightforward generalizations). The quenched particles have a one-body density distribution, $\rho_{0}(\mathbf{r})$, which is obtained by minimizing (15) using the one component (0) version of (23). The density distributions of the remaining (annealed) species of particles are obtained by minimizing the grand potential functional: 


$$
\begin{aligned}
\Omega_{12}^{\mathrm{var}}\left[\left\{\rho_{a}(\mathbf{r})\right\}\right]= & \mathcal{F}_{1}^{\mathrm{id}}\left[\rho_{1}(\mathbf{r})\right]+\mathcal{F}_{2}^{\mathrm{id}}\left[\rho_{2}(\mathbf{r})\right] \\
& +\frac{1}{2} \sum_{n, m=1}^{2} \int \mathrm{d} \mathbf{r} \int \mathrm{d} \mathbf{r}^{\prime} \rho_{n}(\mathbf{r}) \rho_{m}\left(\mathbf{r}^{\prime}\right) v_{n m}\left(\left|\mathbf{r}-\mathbf{r}^{\prime}\right|\right) \\
& +\sum_{n=1}^{2} \int \mathrm{d} \mathbf{r} \int \mathrm{d} \mathbf{r}^{\prime} \rho_{n}(\mathbf{r}) \rho_{0}\left(\mathbf{r}^{\prime}\right) v_{0 n}\left(\left|\mathbf{r}-\mathbf{r}^{\prime}\right|\right) \\
& -\sum_{n=1}^{2} \int \mathrm{d} \mathbf{r} \rho_{n}(\mathbf{r})\left[\mu_{n}-V_{n}^{\mathrm{ext}}(\mathbf{r})\right] .
\end{aligned}
$$

Expressing the density distribution of the quenched particles as $\rho_{0}(\mathbf{r})=\rho_{0}^{b}+\Delta \rho_{0}(\mathbf{r})$, i.e., as a modulation $\Delta \rho_{0}(\mathbf{r})$ around a constant density $\rho_{0}^{b}$, (25) can be written as

$$
\begin{aligned}
\Omega_{12}^{\mathrm{var}}\left[\left\{\rho_{a}(\mathbf{r})\right\}\right]= & \mathcal{F}_{1}^{\mathrm{id}}\left[\rho_{1}(\mathbf{r})\right]+\mathcal{F}_{2}^{\mathrm{id}}\left[\rho_{2}(\mathbf{r})\right]+\mathcal{F}^{\mathrm{ex}}\left[\left\{\rho_{n}(\mathbf{r})\right\}\right] \\
& -\sum_{n=1}^{2} \int \mathrm{d} \mathbf{r} \rho_{n}(\mathbf{r})\left[\mu_{n}^{\mathrm{eff}}-V_{n}^{\mathrm{eff}}(\mathbf{r})\right]
\end{aligned}
$$

where the effective chemical potentials are

$$
\mu_{n}^{\text {eff }}=\mu_{n}-\rho_{0}^{b} \hat{v}_{0 n}(0),
$$

and $\hat{v}_{0 n}(0) \equiv \int \mathrm{d} \mathbf{r} v_{0 n}(r)=\pi^{3 / 2} \epsilon_{0 n} R_{0 n}^{3}$ is the $k \rightarrow 0$ limit of the Fourier transform of the pair potential $v_{0 n}(r)$. The effective external potentials are

$$
V_{n}^{\mathrm{eff}}(\mathbf{r})=V_{n}^{\mathrm{ext}}(\mathbf{r})+\int \mathrm{d} \mathbf{r}^{\prime} \Delta \rho_{0}\left(\mathbf{r}^{\prime}\right) v_{0 n}\left(\left|\mathbf{r}-\mathbf{r}^{\prime}\right|\right)
$$

This analysis demonstrates that the quenched matrix has a very similar effect to that of an external potential acting on the mobile components. However, because of the convolution form in Eq. (28), the matrix effective potentials $V_{n}^{\text {eff }}(\mathbf{r})$ are quite different from the external potentials that are typically tackled within DFT, such as the hard potentials modeling container walls.

\section{A MATRIX OF UNIFORM DENSITY}

As a prerequisite for our subsequent interface study we consider the case where the matrix density profile is constant: $\rho_{0}(\mathbf{r})=\rho_{0}^{b}\left[\right.$ i.e., $\left.\Delta \rho_{0}(\mathbf{r})=0\right]$. In the particular case where any external potentials also vanish, the one body densities of the annealed species are constant: $\rho_{n}(\mathbf{r})=\rho_{n}^{b}, n=1,2$. As convenient variables we use the total (bulk) density of the annealed species, $\rho^{b}=\rho_{1}^{b}+\rho_{2}^{b}$, and the relative concentration, $x$ $\equiv \rho_{2}^{b} / \rho^{b}$. From Eq. (25) the Helmholtz free energy per particle, $f_{12}=F / N$, for the annealed GCM fluid is simply [21]

$$
f_{12}\left(\rho^{b}, x\right)=f_{12}^{\mathrm{id}}\left(\rho^{b}, x\right)+\frac{1}{2} \rho^{b} \hat{V}_{0}(x)+\rho_{0}^{b} Q(x),
$$

where $f_{12}^{\mathrm{id}}$ is the ideal gas contribution,

$$
\begin{aligned}
f_{12}^{\mathrm{id}}\left(\rho^{b}, x\right)= & (1-x) \ln (1-x)+x \ln x+(1-x) \ln \left(\Lambda_{1}^{3} \rho^{b}\right) \\
& +x \ln \left(\Lambda_{2}^{3} \rho^{b}\right)-1,
\end{aligned}
$$

the interaction term is

$$
\hat{V}_{0}(x)=(1-x)^{2} \hat{v}_{11}(0)+2 x(1-x) \hat{v}_{12}(0)+x^{2} \hat{v}_{22}(0),
$$

and the contribution due to the matrix is

$$
Q(x)=(1-x) \hat{v}_{10}(0)+x \hat{v}_{20}(0) .
$$

The latter has a particularly simple form; it is independent of $\rho^{b}$ and only linearly dependent on $x$. This leads to several special features of the fluid inside the matrix. First, the pressure of the annealed GCM fluid in the matrix is independent of the matrix density $\rho_{0}^{b}$. As the pressure $P$ is obtained from

$$
P=-\left(\frac{\partial f_{12}}{\partial v}\right)_{x, T},
$$

where $v=1 / \rho^{b}$ is the volume per particle and the matrix contribution to the free energy (29) is independent of $\rho^{b}$, it follows that $P$ is independent of $\rho_{0}^{b}$.

Second, the phase boundaries in the $\left(x, \rho^{b}\right)$ plane of the fluid inside the matrix are the same as those without the matrix (it is known that the binary GCM fluid can exhibit fluid-fluid phase separation [20,21]). This can be seen as follows: the locus of the spinodal line can be obtained from the Gibbs free energy per particle, $g_{12}=f_{12}+P v$, via the condition

$$
\left(\frac{\partial^{2} g_{12}}{\partial x^{2}}\right)_{P, T}=0 .
$$

Since $P$ is independent of $\rho_{0}^{b}$ and the terms in $f_{12}$ (and therefore in $g_{12}$ ) that are functions of $\rho_{0}^{b}$ are only linearly dependent on $x$, we see from (34) that in the $\left(x, \rho^{b}\right)$ plane the spinodal line will be the same for all values of $\rho_{0}^{b}$. The binodal (coexistence curve) is obtained from the conditions of equal pressure $P$ and equal chemical potentials in the coexisting phases,

$$
\begin{aligned}
P_{\alpha} & =P_{\beta}, \\
\mu_{1, \alpha} & =\mu_{1, \beta}, \\
\mu_{2, \alpha} & =\mu_{2, \beta},
\end{aligned}
$$

where $\alpha$ and $\beta$ label the coexisting phases. From Eq. (27), we see that the presence of the matrix results in only a linear shift in the chemical potentials of the fluid particles, and this is the same in both fluid phases, $\alpha$ and $\beta$. Therefore, the binodal curve for the binary fluid in the matrix is at the same location in the $\left(x, \rho^{b}\right)$ plane as it is without the matrix.

The special form of the free energy makes for a simple analysis of the situation when one has a bulk binary GCM fluid acting as a reservoir which is and coupled to the system inside the matrix that consists of quenched particles of a third species of GCM particles. The chemical potentials, $\mu_{1}$ and $\mu_{2}$, are set by the fluid in the bulk reservoir. One can calculate $\rho_{1}^{b}$ and $\rho_{2}^{b}$, the densities of the fluid in the matrix, 
using Eq. (27), i.e., the densities of the fluid in the matrix are simply those of a bulk fluid with chemical potentials equal to $\mu_{n}^{\text {eff }}, n=1,2$, given by Eq. (27).

For a binary GCM fluid exhibiting phase separation, this means that the chemical potentials, $\mu_{n}$, can correspond to a fluid on one side of the binodal of the reservoir (say, the $\alpha$-phase), but the effective chemical potentials $\mu_{n}^{\text {eff }}$, given by Eq. (27), for the fluid inside the matrix can correspond to the other side of the binodal (the $\beta$-phase). Under these circumstances a (capillary) condensation of the coexisting $\beta$ phase occurs in the matrix. Such condensation transitions are well known for fluids in porous media (the matrix) $[1,2,5-8]$. Perhaps the best known case is that of a gas condensing to a liquid at pressures below the bulk saturated vapor pressure or, for fixed pressure, at temperatures above the bulk boiling temperature. In this case the bulk $\alpha$ phase corresponds to the gas and the $\beta$ phase to the liquid [36].

Owing to the simplicity of the present model fluid and the theoretical description that we use, Eq. (27) provides a very simple mapping between points in the $\left(x, \rho^{b}\right)$ plane corresponding to the density and concentration of the fluid in the bulk reservoir and the same quantities for the fluid in the matrix. For a given matrix density (and given parameters of the pair potential between matrix and fluid particles) we can easily determine the matrix condensation phase behavior.

\section{RESULTS FOR A PARTICULAR BINARY MIXTURE}

We consider cases where the annealed fluid components are characterized by the pair potential parameters $\epsilon_{11}=\epsilon_{22}$ $=2 k_{B} T, \epsilon_{12}=1.8877 k_{B} T, R_{22}=0.665 R_{11}$; this implies from Eq. (21) that $R_{12}=0.849 R_{11}$. This particular binary mixture exhibits bulk fluid-fluid phase separation and was investigated in a number of previous studies [21,24-27]. The bulk phase diagram is displayed in Fig. 1. The parameters chosen for the pair potentials characterizing matrix particles are $\epsilon_{00}=2 k_{B} T$, $\epsilon_{01}=k_{B} T, \epsilon_{02}=0.8 k_{B} T, R_{00}=7 R_{11}$; from Eq. (21) it follows that $R_{01}=5 R_{11}$ and $R_{02}=4.97 R_{11}$. The reason for this choice of parameters is that much is known about the behavior of an annealed fluid of such (big) particles dissolved in the binary (solvent) fluid [25-27]; hence we can make connections with that body of work. In Fig. 1(b) we display a portion of the phase diagram, together with the loci in the reservoir phase diagram of the state points which correspond [via Eq. (27)] to points on the binodal/spinodal for the fluid adsorbed in the matrix. We display results for three densities of the quenched particles, $\rho_{0}^{b} R_{11}^{3}=0.0001,0.001$, and 0.002 (or, equivalently, $\rho_{0}^{b} R_{00}^{3}=0.0343,0.343$, and 0.686$)$. As the matrix density $\rho_{0}^{b}$ is increased, the binodal for the fluid adsorbed in the matrix shifts to larger $x$, away from the reservoir binodal. Note that one would obtain the same shift for a matrix with a different set of pair potential parameters and matrix densities, so long as the products $\rho_{0}^{b} \hat{v}_{0 n}(0)$ remain the same [recall Eq. (27)]. For the above set of matrix pair potential parameters we know [25-27] a single big particle can be surrounded by a thick adsorbed film of the coexisting phase. Furthermore, on decreasing $x$, for certain fixed total fluid densities, there can be a transition from a thin to a thick adsorbed film (see Fig.
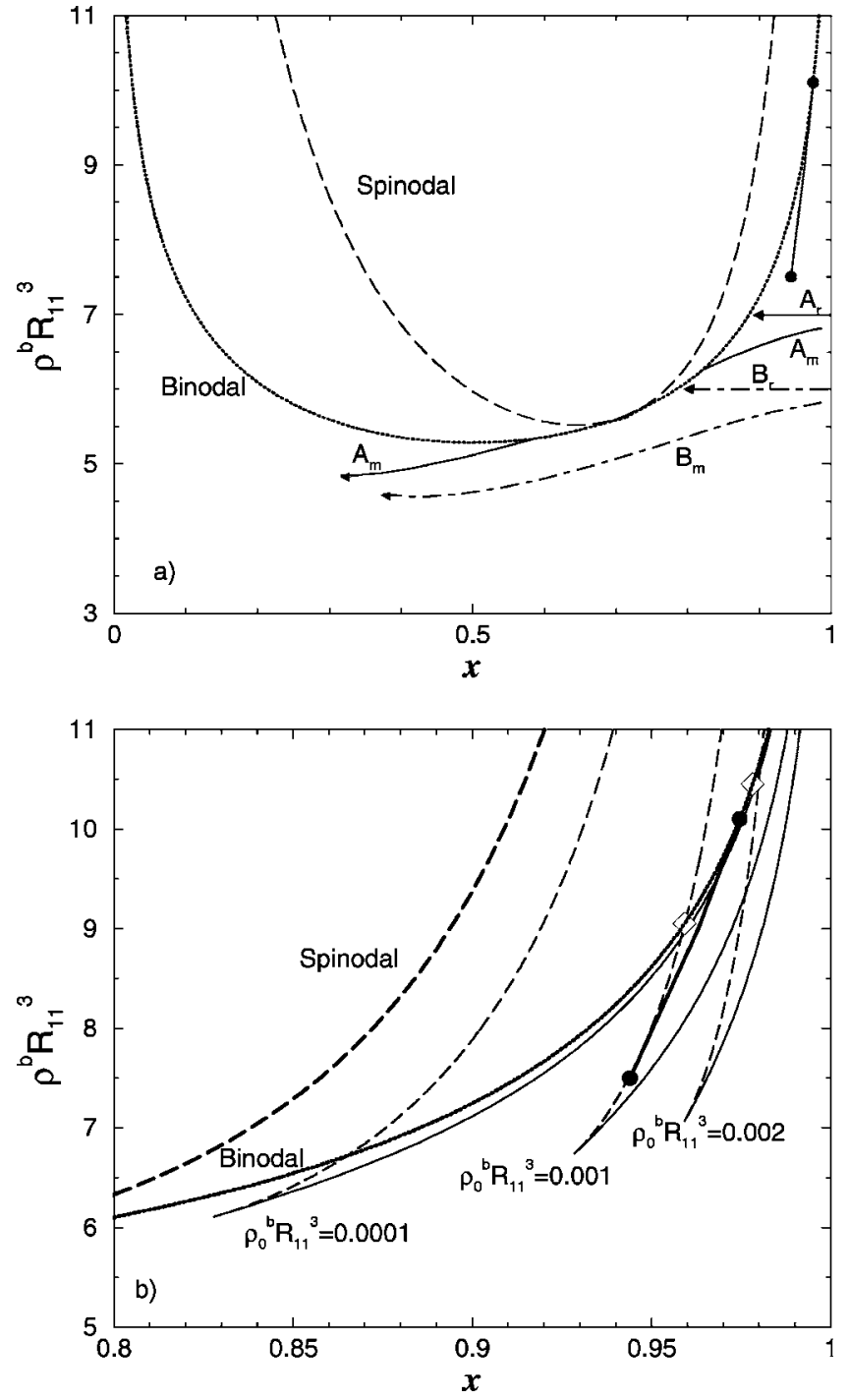

FIG. 1. The bulk phase diagram for a binary mixture of GCM particles with $\epsilon_{12} / \epsilon_{11}=0.944$ and $R_{22} / R_{11}=0.665$ (see also Ref. [21]) in terms of the total density, $\rho^{b}$, and the concentration, $x$, of the smaller species 2 . In (a) the paths $A_{r}$ and $B_{r}$ (arrows) are at constant total density in the reservoir. The paths $A_{m}$ and $B_{m}$ indicate the corresponding paths for the fluid in a matrix of density $\rho_{0}^{b} R_{11}^{3}=10^{-3}$. (b) shows an expanded view of the phase diagram. The three additional pairs of binodal (solid line) and spinodal (dashed) lines show the loci in the reservoir phase diagram which correspond [via Eq. (27)] to the binodal/spinodal of the fluid adsorbed in the matrix, plotted for three different matrix densities. In (a) and (b) the solid line whose ends are denoted by filled circles is the thin-thick adsorbed film transition of the binary fluid adsorbed around a single big GCM particle with pair potential parameters $\epsilon_{01}=k_{B} T$, $\epsilon_{02}=0.8 k_{B} T, R_{01} / R_{11}=5.0$, and $R_{02} / R_{11}=4.97$-see Refs. [26,27]. The two points $(\diamond)$ are where the spinodal for this single-particle thin-thick adsorbed transition meets the bulk fluid binodal.

1). Given this information, and the typical size of the (thick) adsorbed film, one would expect the matrix condensation line [e.g. those in Fig. 1(b)] to be located in the vicinity of the single-particle thin-thick adsorbed film transition line, provided the density of the quenched matrix particles is sufficiently high that their adsorbed films (start to) overlap. 


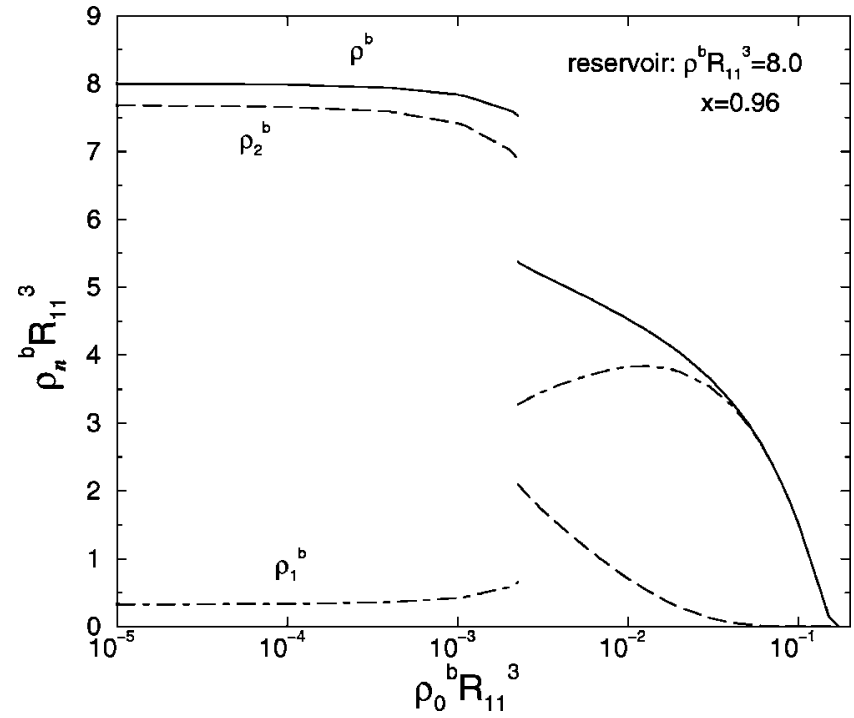

FIG. 2. Fluid densities, $\rho_{n}^{b}$, inside the matrix corresponding to a reservoir with fixed total density $\rho^{b} R_{11}^{3}=8.0$ and concentration $x=0.96$ plotted versus (uniform) matrix density $\rho_{0}^{b}$. For $\rho_{0}^{b} R_{11}^{3}>2.3 \times 10^{-3}$, condensation of the coexisting fluid phase occurs in the matrix. Note that $\rho_{1}^{b}$ is a nonmonotonic function of $\rho_{0}^{b}$, whereas $\rho_{2}^{b}$ and the total density $\rho^{b}$ are monotonically decreasing functions of $\rho_{0}^{b}$.

Near bulk coexistence, the adsorbed film around a single big particle has a radius $\sim 7 R_{11}[25-27]$. One would therefore expect the matrix condensation line to be near the singleparticle thin-thick adsorbed film transition line when the matrix density $\rho_{0}^{b} \simeq\left(4 \pi\left(7 R_{11}\right)^{3} / 3\right)^{-1}=0.7 \times 10^{-3} R_{11}^{-3}$. This is indeed what we observe. In fact, we find that the spinodal line for the single-particle thin-thick transition line lies very close to the spinodal line (in the reservoir phase diagram) for a matrix of density $\rho_{0}^{b}=10^{-3} R_{11}^{-3}$ [see Fig. 1(b)]. This observation provides physical insight into the mechanism driving the condensation in the matrix: condensation occurs when the big matrix particles are sufficiently close that the adsorbed films around each of them can overlap. This means that condensation in the present soft core system is somewhat different from the condensation that we alluded to earlier where the condensation occurs in the gaps in the matrix, i.e., as a surface effect, where the films on (opposite) matrix surfaces join. Rather, for the present soft-core system, the onset of condensation is related to the density profile of the solvent around and inside a single matrix particle. One can therefore determine much about the fluid behavior in the bulk matrix from knowing the behavior around a single matrix particle.

In Fig. 2 we display the fluid densities, $\rho_{n}^{b}, n=1,2$, inside a matrix of quenched particles with uniform density for the case of a bulk fluid reservoir with total density $\rho^{b} R_{11}^{3}=8.0$ and concentration $x=0.96$. Results are plotted for a range of matrix densities $\rho_{0}^{b}$. For $\rho_{0}^{b} R_{11}^{3}>2.3 \times 10^{-3}$, we find condensation of the coexisting fluid phase in the matrix. We also find that $\rho_{1}^{b}$ (dashed-dotted line) is a nonmonotonic function of $\rho_{0}^{b}$, whereas $\rho_{2}^{b}$ (dashed line) and the total density $\rho^{b}=\rho_{1}^{b}+\rho_{2}^{b}$ (solid line) are monotonically decreasing functions of $\rho_{0}^{b}$. For $\rho_{0}^{b} R_{11}^{3} \geq 0.1$ the fluid densities are very small; both species are expelled from the matrix. This behavior is typical of that found for reservoir state points lying on the right-hand side of the bulk binodal in Fig. 1.

\section{INHOMOGENEOUS FLUID DENSITY PROFILES: PLANAR INTERFACE}

In this section we determine the inhomogeneous fluid density profiles of the binary fluid for a simple model of an interface in which the quenched matrix particles are described by an average density distribution:

$$
\rho_{0}(z)=\left\{\begin{array}{cc}
\rho_{0}^{b}, & z \leqslant 0, \\
0, & z>0,
\end{array}\right.
$$

i.e., the matrix particles are confined to the half-space with (Cartesian) coordinate $z \leqslant 0$ and the fluid reservoir is at $z>0$. The planar interface is located at $z=0$, where $z$ is the coordinate perpendicular to the interface. The step function in Eq. (36) is not the most realistic model for the matrix density profile; one would expect some structure in the profile for $z \rightarrow 0^{-}$that will depend on how the quenched matrix is formed. However, the simple choice (36) allows us to explore some of the interfacial phenomena that can be exhibited by the present system. We do not expect our results to be changed qualitatively were we to chose a more realistic profile.

In Fig. 3 we display the density profiles $\rho_{n}(z), n=1,2$, of the binary GCM fluid for cases where the reservoir fluid has a fixed bulk density $\rho^{b} R_{11}^{3}=7.0$ and concentration $x=0.888$ that is near to coexistence (located at $x_{\text {coex }}=0.88544$ ). The density profiles are calculated for six matrix densities $\rho_{0}^{b} R_{11}^{3}=10^{-5}, 10^{-4}, 10^{-3}, 10^{-2}, 0.05$, and 0.1 . Provided the matrix density is sufficiently high the coexisting fluid condenses in the matrix. For large values of the matrix density the fluid is expelled from the bulk of the matrix-observe the results for $\rho_{0}^{b} R_{11}^{3}=0.1$. The densities of the fluid in the bulk of the matrix $(z \rightarrow-\infty)$ behave in a similar manner to the results displayed in Fig. 2. Strikingly, in addition to condensation in the bulk of the matrix, a wetting film of the coexisting phase develops on the surface of the matrix. This film is present in spite of the fact that the fluid density in the matrix is very low, owing to the high matrix density. Similar wetting behavior was found for the present GCM fluid mixture at a planar wall, for several choices of (repulsive) wall-fluid potentials [24].

Since the decay of the effective matrix potential (28) into the bulk of the reservoir fluid is short ranged, the thickness $l$ of the wetting film of the coexisting phase on the surface of the matrix (i.e., $l$ is the distance of the wetting film interface from $z=0$ ) should increase as $l \sim-\xi_{w} \ln (\delta x)$, where $\delta x=x-x_{\text {coex }}$ is the difference in concentration between the reservoir concentration $x$ and the concentration at bulk coexistence $x_{\text {coex }} . \xi_{w}$ is the true correlation length in the bulk coexisting phase which is wetting the wall (matrix)-reservoir interface [24].

We investigate the wetting behavior in more detail in Figs. 4 and 5 where we display the density profiles of both species for the binary fluid at the planar interface given by Eq. (36) with fixed $\rho_{0}^{b} R_{11}^{3}=10^{-3}$. Results are presented for 

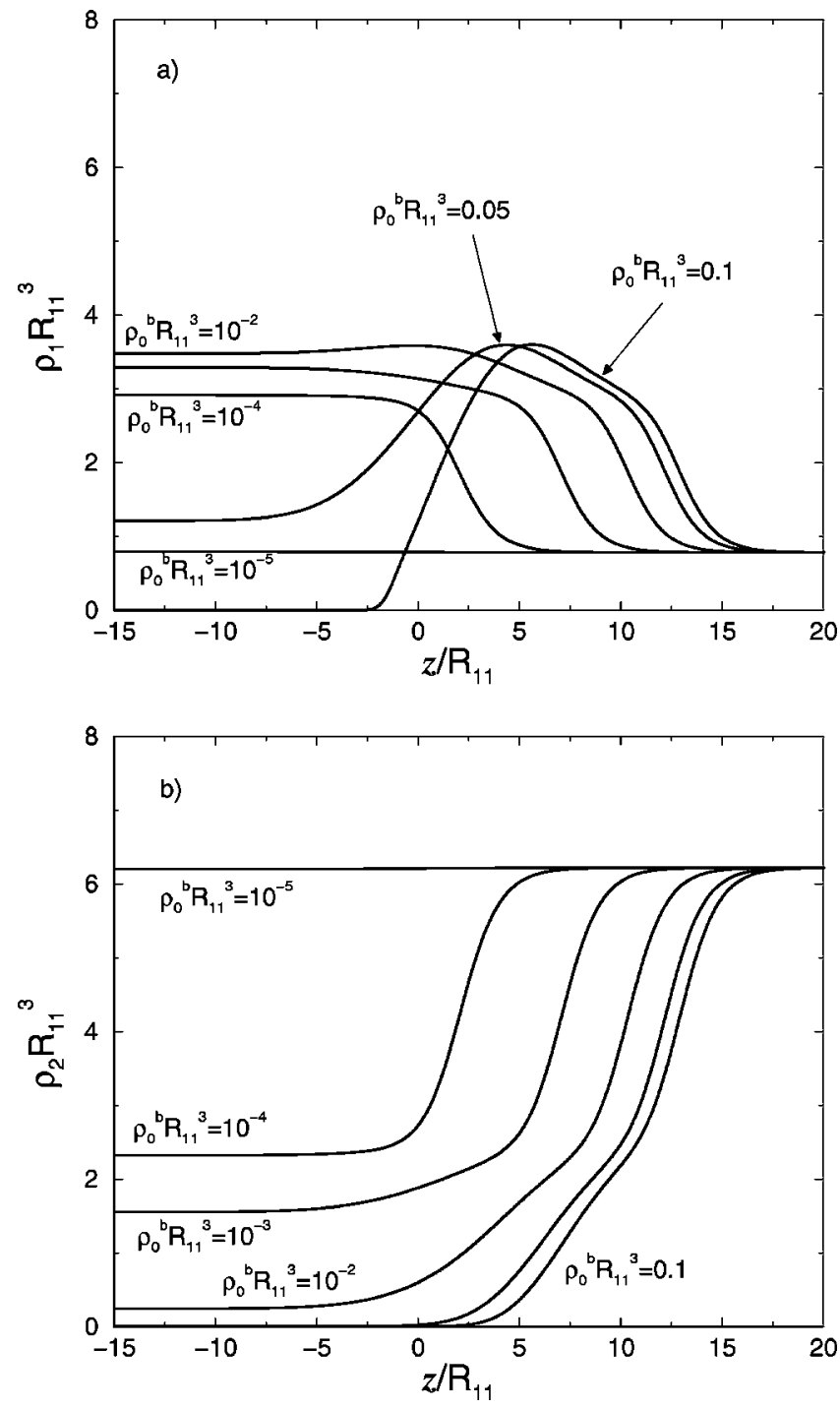

FIG. 3. Density profiles of the binary GCM fluid at the planar interface between a matrix with density profile given by Eq. (36) and a reservoir of fluid (at $z>0$ ) with fixed total bulk density $\rho^{b} R_{11}^{3}=7.0$ and concentration $x=0.888$. The density profiles for species 1 are shown in (a) and those for species 2 are in (b). The density profiles are calculated for matrix bulk densities $\rho_{0}^{b} R_{11}^{3}=10^{-5}, 10^{-4}, 10^{-3}, 10^{-2}, 0.05$, and 0.1 . Note that for intermediate densities the coexisting fluid phase is condensed in the matrix. At this reservoir state point, there is a wetting film extending from the interface at $z=0$ into the bulk reservoir fluid for the higher matrix densities.

reservoir state points corresponding to points along paths $A_{r}$ and $B_{r}$ in Fig. 1(a). In Fig. 4 the profiles are calculated along path $A_{r}\left(\rho^{b} R_{11}^{3}=7\right)$ for a range of concentrations approaching the reservoir binodal. Between $x=0.937$ and 0.934 the coexisting phase condenses in the matrix - note the jump in the profiles. As $x$ is decreased further, a wetting film develops on the surface of the matrix. This increases in extent as $x$ approaches its value at coexistence. In Fig. 5 we display the density profiles for the same matrix density but now for reservoir total density $\rho^{b} R_{11}^{3}=6.0$, corresponding to path $B_{r}$ in Fig. 1(a). Once again the profiles are calculated for a range
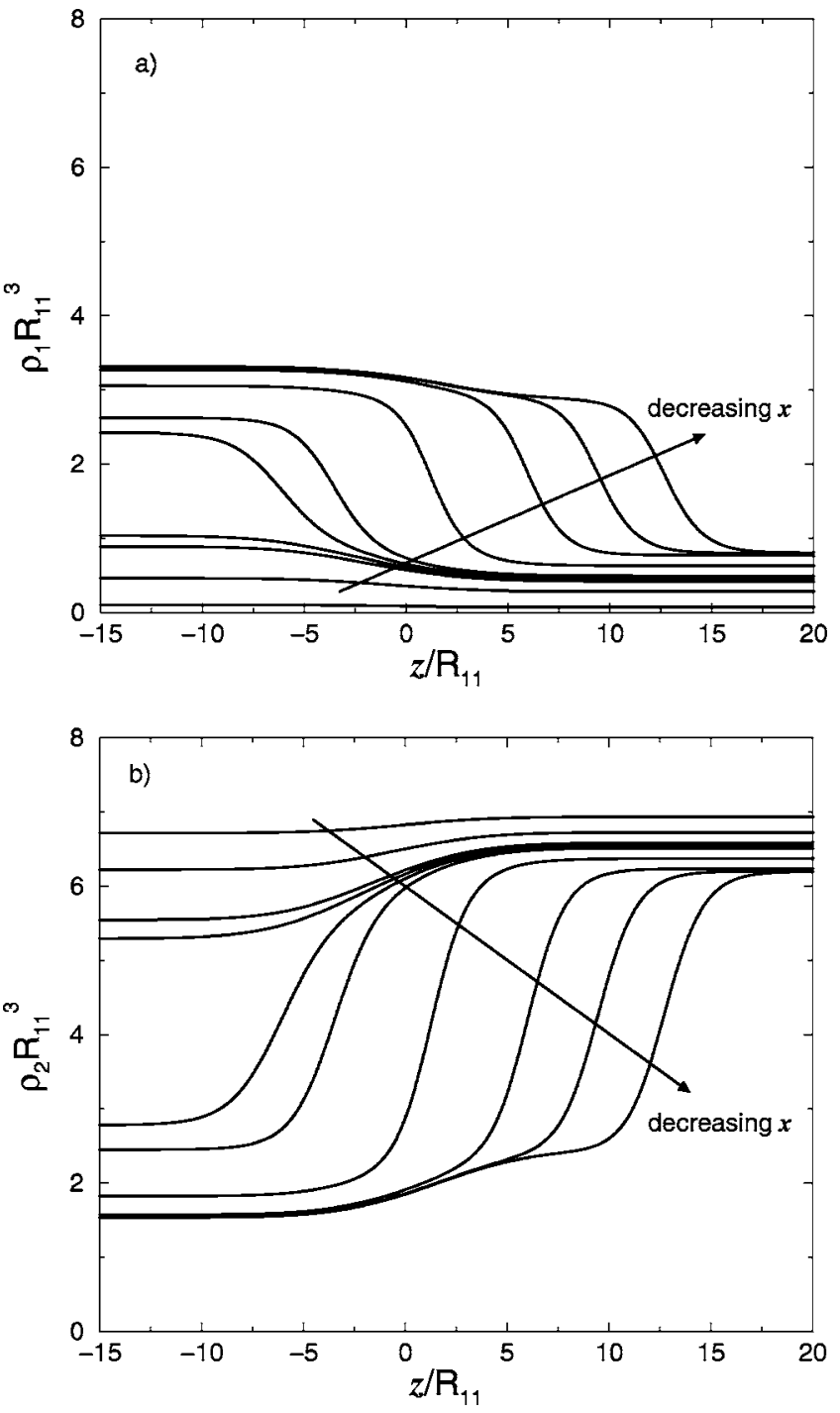

FIG. 4. Density profiles of the binary fluid at the planar interface between a matrix with density profile given by Eq. (36), with $\rho_{0}^{b} R_{11}^{3}=10^{-3}$. The bulk fluid reservoir $(z \rightarrow \infty)$ has fixed total density $\rho^{b} R_{11}^{3}=7.0$. The profiles are calculated for concentrations $x=0.99$, 0.96, 0.94, 0.937, 0.934, 0.93, 0.91, 0.89, 0.886, and 0.88545 (bulk coexistence is at $\left.x_{\text {coex }}=0.88544\right)$. These correspond to a series of points along path $A_{r}$ in Fig. 1(a). Note that between $x=0.937$ and 0.934 , condensation of the coexisting phase occurs in the matrix. Decreasing $x$ further, a wetting film develops on the surface of the matrix.

of concentrations approaching the reservoir binodal and we observe a wetting film developing on the surface of the matrix. Note the development of a shoulder in the profile for $x=0.785$, close to coexistence. Contrary to the case in Fig. 4, the density of the fluid adsorbed in the matrix changes continuously as $x$ is varied; there is no jump as path $B_{r}$ lies below the critical point of the matrix condensation binodal for $\rho_{0}^{b} R_{11}^{3}=10^{-3}$ see Fig. 1(b). In both cases the wetting film thickens as $x \rightarrow x_{\text {coex }}$, and we find that the film thickness $l$ $\sim-\xi_{w} \ln (\delta x)$. In general, when the coexisting phase is condensed in the matrix and the reservoir state point is near to coexistence we expect the matrix-reservoir interface to be 

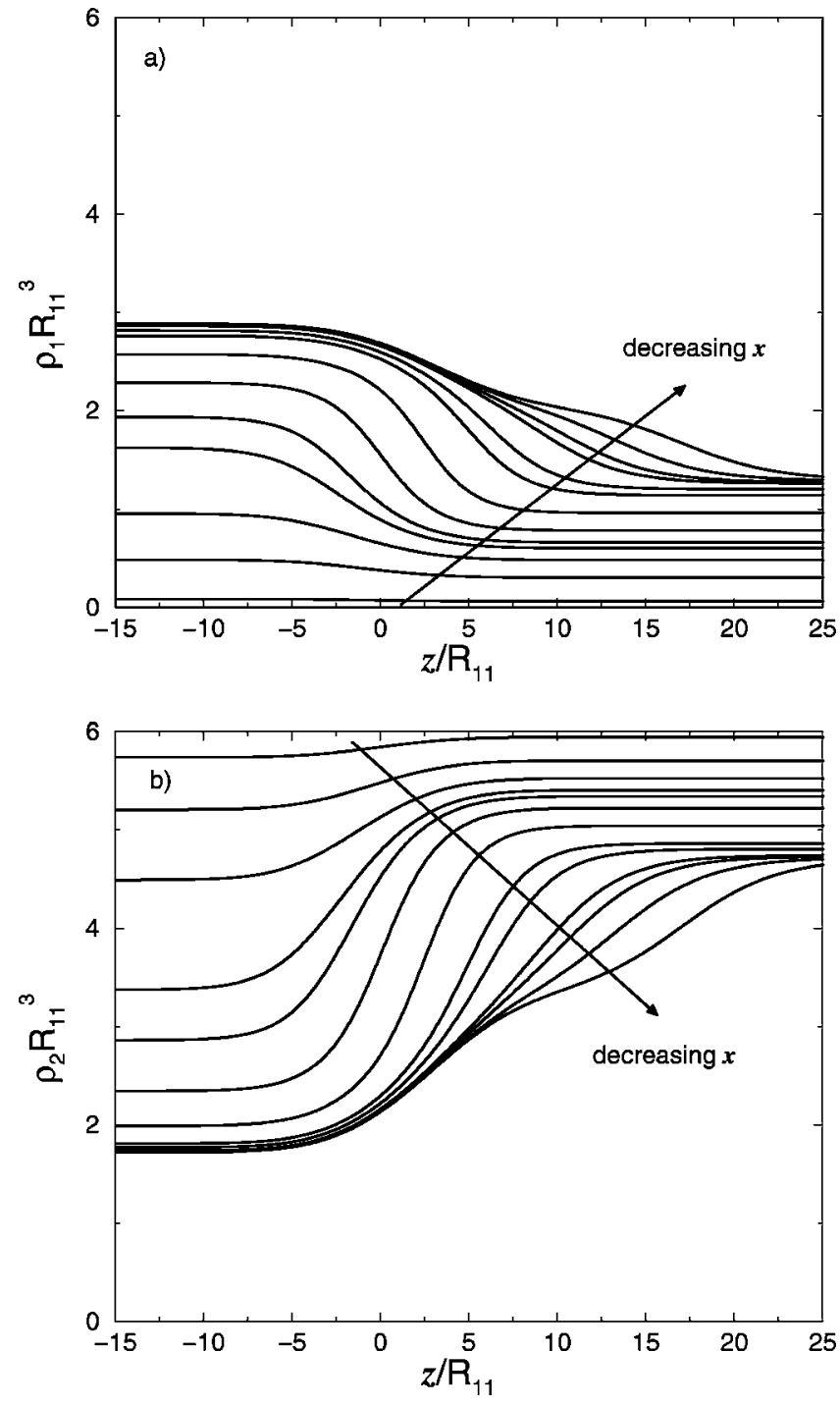

FIG. 5. Density profiles of the binary fluid at the planar interface between a matrix with density profile given by Eq. (36), with $\rho_{0}^{b} R_{11}^{3}=10^{-3}$. The bulk fluid reservoir $(z \rightarrow \infty)$ has total density $\rho^{b} R_{11}^{3}=6.0$. The profiles are calculated for concentrations $x$ $=0.99,0.95,0.92,0.9,0.89,0.87,0.84,0.81,0.8,0.79,0.788$, 0.786 , and 0.785 (bulk coexistence is at $x_{\text {coex }}=0.7848$ ). These correspond to a series of points along path $B_{r}$ in Fig. 1(a). For decreasing $x$, a wetting film develops on the surface of the matrix. Note that, contrary to the case in Fig. 4, the density of the fluid adsorbed in the matrix changes continuously with $x$.

wet completely by a film of the coexisting phase as $x$ $\rightarrow x_{\text {coex }}$.

\section{CONCLUSIONS}

We have considered the properties of a ternary GCM mixture, one of whose species is quenched, modeling a rigid matrix of (crosslinked) polymers. The two annealed species then model a binary polymer solution that is immersed in the matrix. Using a DFT treatment we have calculated both the behavior of the binary mixture in the bulk matrix and its properties at a planar interface, described by the profile (36), between the matrix and a reservoir of the fluid.

In the first case where the fluid is adsorbed in the bulk matrix with uniform density $\rho_{0}^{b}$, we find that in the total density versus concentration $\left(x, \rho^{b}\right)$ representation of the adsorbed fluid phase diagram, the location of the fluid-fluid binodal is independent of $\rho_{0}^{b}$. This somewhat surprising result is a direct consequence of the soft core nature of both the adsorbed fluid and matrix particles as described within a second-order (in densities) free energy — see Eq. (29). In particular the contribution due to the matrix is independent of the density of the annealed fluid. For given fluid densities in the reservoir, the calculation of the fluid densities in the matrix is very straightforward, and mapping out lines of (capillary) condensation in the matrix, in the $\left(x, \rho^{b}\right)$ phase diagram, is readily performed.

Applying a Helmholtz free energy functional more accurate than the simple RPA functional (23), we would expect the fluid phase diagram in the $\left(x, \rho^{b}\right)$ plane to depend explicitly on the matrix density $\rho_{0}^{b}$. However, bearing in mind the accuracy of (23) at high fluid densities [18-23], we expect to find only a weak dependence on $\rho_{0}^{b}$ in the true phase diagram of the binary GCM immersed in a GCM matrix.

In earlier work [25-27], we found that due to the soft-core nature of the particles, condensation of the coexisting phase occurs both on the surface and inside a single big particle. Making connection with this work, here we found that for matrices composed of big GCM particles with density $\rho_{0} R_{00}^{3} \sim 1$ (i.e., densities near the overlap concentration), the mechanism for capillary condensation of the coexisting fluid phase in the matrix is the joining of the thick films of the coexisting phase that form around individual big GCM particles as bulk coexistence is approached [25-27]. This mechanism is somewhat different from what occurs in hard core systems, where the wetting films grow on the surface of big matrix particles and condensation occurs throughout the matrix by the joining of these surface films.

Much of the simplicity inherent in the present theory stems from the fact that the effect of the matrix on the fluid can be described in terms of a shift in the effective chemical potential [cf Eq. (27)] and an effective one body external potential-see Eq. (28). Note that this simplification is not clear at the outset: a particular configuration of matrix particles can, of course, be treated as an external potential for the adsorbed fluid, but having averaged over the ensemble of possible matrix configurations, the description of the matrix then enters on the level of the (ensemble average) one body density profile of the matrix particles. That this problem can be subsequently mapped to an effective one body potential, which is much simpler to treat than the potential exerted by a particular configuration of matrix particles, makes determining properties of the inhomogeneous fluid in contact with an inhomogeneous matrix tractable. Following this procedure we were able to calculate adsorption and wetting behavior of the binary mixture on the surface of the matrix in contact with a reservoir of the fluid. In the case of a reservoir at state points near to coexistence, we found a rich interplay between the fluid condensation in the bulk of the matrix and wetting behavior at the interface. We conclude that the present DFT approach constitutes a powerful approach for 
treating fluids in inhomogeneous random media. Although the GCM model is a very simple one, it is physically realistic and the results obtained from analyzing its properties should provide much insight into bulk and interfacial phenomena of fluids adsorbed in random porous media.

\section{ACKNOWLEDGMENTS}

A.J.A. acknowledges the support of EPSRC under Grant No. GR/S28631/01. M.S. acknowledges the support of the SFB TR6 "Colloidal dispersions in external fields" of the DFG.
[1] R. Evans, J. Phys.: Condens. Matter 2, 8989 (1990).

[2] L. D. Gelb, K. E. Gubbins, R. Radhakrishnan, and M. Sliwinska-Bartkowiak, Rep. Prog. Phys. 62, 1573 (1999).

[3] V. Talanquer and D. W. Oxtoby, J. Chem. Phys. 113, 7013 (2000).

[4] D. Woywod and M. Schoen, Phys. Rev. E 67, 026122 (2003) and references therein.

[5] E. Kierlik, P. A. Monson, M. L. Rosinberg, L. Sarkisov, and G. Tarjus, Phys. Rev. Lett. 87, 055701 (2001).

[6] E. Kierlik, P. A. Monson, M. L. Rosinberg, and G. Tarjus, J. Phys.: Condens. Matter 14, 9295 (2002).

[7] L. Sarkisov and P. A. Monson, Phys. Rev. E 65, 011202 (2002).

[8] F. Detcheverry, E. Kierlik, M. L. Rosinberg, and G. Tarjus, Phys. Rev. E 68, 061504 (2003).

[9] H. J. Woo and P. A. Monson, Phys. Rev. E 67, 041207 (2003).

[10] M. Schmidt, L. Lafuente, and J. A. Cuesta, J. Phys.: Condens. Matter 15, 4695 (2003).

[11] W. G. Madden and E. D. Glandt, J. Stat. Phys. 51, 537 (1988).

[12] J. A. Given and G. Stell, J. Chem. Phys. 97, 4573 (1992).

[13] R. Evans, Adv. Phys. 28, 143 (1979).

[14] R. Evans, in Fundamentals of Inhomogeneous Fluids, edited by D. Henderson (Dekker, New York, 1992), Chap. 3, p. 85.

[15] M. Schmidt, Phys. Rev. E 66, 041108 (2002).

[16] H. Reich and M. Schmidt, J. Stat. Phys. 116, 1683 (2004).

[17] P. P. F. Wessels, M. Schmidt, and H. Löwen, Phys. Rev. Lett. 94, 078303 (2005) and references therein.

[18] C. N. Likos, Phys. Rep. 348, 267 (2001).

[19] A. Lang, C. N. Likos, M. Watzlawek, and H. Löwen, J. Phys.: Condens. Matter 12, 5087 (2000).

[20] A. A. Louis, P. G. Bolhuis, and J. P. Hansen, Phys. Rev. E 62, 7961 (2000).

[21] A. J. Archer and R. Evans, Phys. Rev. E 64, 041501 (2001).
[22] A. J. Archer, C. N. Likos, and R. Evans, J. Phys.: Condens. Matter 16, L297 (2004).

[23] A. Patrykiejew, O. Pizio, and S. Sokolowski, Mol. Phys. 102, 801 (2004).

[24] A. J. Archer and R. Evans, J. Phys.: Condens. Matter 14, 1131 (2002).

[25] A. J. Archer, R. Evans, and R. Roth, Europhys. Lett. 59, 526 (2002).

[26] A. J. Archer and R. Evans, J. Chem. Phys. 118, 9726 (2003).

[27] A. J. Archer, R. Evans, R. Roth, and M. Oettel, J. Chem. Phys. 122, 084513 (2005).

[28] M. Schmidt, E. Schöll-Paschinger, J. Köfinger, and G. Kahl, J. Phys.: Condens. Matter 14, 12099 (2002).

[29] J. Dautenhahn and C. K. Hall, Macromolecules 27, 5399 (1994).

[30] A. A. Louis, P. G. Bolhuis, J. P. Hansen, and E. J. Meijer, Phys. Rev. Lett. 85, 2522 (2000).

[31] P. G. Bolhuis, A. A. Louis, J. P. Hansen, and E. J. Meijer, J. Chem. Phys. 114, 4296 (2001).

[32] A. A. Louis, P. G. Bolhuis, R. Finken, V. Krakoviack, E. J. Meijer, and J.-P. Hansen, Physica A 306, 251 (2002).

[33] P. G. Bolhuis and A. A. Louis, Macromolecules 35, 1860 (2002).

[34] C. N. Likos, S. Rosenfeldt, N. Dingenouts, M. Ballauff, P. Lindner, N. Werner, and F. Vögtle, J. Chem. Phys. 117, 1869 (2002).

[35] C. N. Likos, M. Schmidt, H. Löwen, M. Ballauf, D. Pötschke, and P. Lindner, Macromolecules 34, 2914 (2001).

[36] For a recent experimental study of argon in porous Vycor, see D. G. Jones and H. M. Fretwell, J. Phys.: Condens. Matter 15, 4709 (2003). This paper provides references to earlier experimental work. 